\title{
WHICH CONNECTED METRIC SPACES ARE COMPACT?
}

\author{
GERALD BEER
}

\begin{abstract}
A metric space $X$ is called chainable if for each $\varepsilon>0$ each two points in $X$ can be joined by an $\varepsilon$-chain. $X$ is called uniformly chainable if for each $\varepsilon$ there exists an integer $n$ such that each two points can be joined by an $\varepsilon$-chain of length at most $n$.

THEOREM. A chainable metric space $X$ is a continum if and only if $X$ is uniformly chainable and there exists $\delta>0$ such that each closed $\delta$-ball is compact.

Using Ramsey's Theorem a sequential characterization of uniformly chainable metric spaces is obtained, paralleling the one for totally bounded spaces.
\end{abstract}

Let $(X, d)$ be a metric space. If $p$ and $q$ are points of $X$ an $\varepsilon$-chain of length $n$ from $p$ to $q$ is a finite sequence $a_{0}, a_{1}, a_{2}, \ldots, a_{n}$ in $X$ such that $a_{0}=p, a_{n}=q$, and $d\left(a_{j-1}, a_{j}\right) \leqslant \varepsilon$ for $j=1, \ldots, n$. We call $X \varepsilon$-chainable if each two points in $X$ can be joined by an $\varepsilon$-chain, and $X$ is called chainable if $X$ is $\varepsilon$-chainable for each positive $\varepsilon$. The chainable spaces include the connected spaces. Moreover, chainability characterizes the connected spaces among the compact ones [2]. The main purpose of this note is to characterize the compact spaces among the connected spaces (more generally the chainable ones). Chainable spaces that are compact satisfy two conditions, one stronger than completeness and the other weaker than total boundedness, two conditions used frequently to characterize compactness when connectivity is irrelevant.

Before proceeding we set forth some notation. Let $X$ be a chainable metric space. If $a \in X$ then $B_{e}[a]$ will denote the closed $\varepsilon$-ball with center $a$. If $A \subset X$, $\cup_{x \in A} B_{e}[x]$ will be designated by $B_{e}[A]$. Inductively construct the set $B_{e}^{n}[A]$ for each $n \in Z^{+}$as follows: $B_{\varepsilon}^{1}[A]=B_{e}[A]$ and for each $n>2$ set $B_{\varepsilon}^{n}[A]=$ $B_{\varepsilon}\left[B_{\varepsilon}^{n-1}[A]\right]$. The following should be observed:

(1) $B_{e}^{n}[A] \subset B_{e}^{n+1}[A]$.

(2) $B_{\varepsilon}^{n}[A] \subset B_{n e}[A]$.

(3) $\cup_{n=1}^{\infty} B_{e}^{n}[A]=X$ if $A \neq \varnothing$.

Finally if $\varepsilon>0$ define $\phi_{\varepsilon}: X \times X \rightarrow\{0,1,2,3, \ldots\}$ by $\phi_{\varepsilon}(x, y)=$ the length of the shortest $\varepsilon$-chain from $x$ to $y$.

Definition. Let $X$ be a chainable metric space. $X$ is called uniformly $\varepsilon$-chainable if there exists a positive integer $n$ such that each two points in $X$ can be joined by an $\varepsilon$-chain of length at most $n$. $X$ is called uniformly chainable if it is uniformly $\varepsilon$-chainable for all positive $\varepsilon$.

Definition. Let $X$ be a metric space. $X$ is called uniformly locally compact if there exists $\varepsilon>0$ for which each closed $\varepsilon$-ball is compact.

Received by the editors April 8, 1980 and, in revised form, April 2, 1981.

AMS (MOS) subject classifications (1970). Primary 54E45, 54D05.

() 1981 American Mathematical Society 0002-9939/81/0000-0578/\$02.25 
LEMMA. Let $X$ be a chainable metric space. If $X$ is totally bounded then $X$ is uniformly chainable. If $X$ is uniformly locally compact then $X$ is complete.

Proof. Suppose $\varepsilon$ is a positive number. If $X$ is totally bounded, then we can find $\left\{x_{1}, \ldots, x_{n}\right\}$ in $X$ such that $X=\cup_{i=1}^{n} B_{e}\left[x_{i}\right]$. It follows that each two points of $X$ can be joined by an $\varepsilon$-chain of length at $\operatorname{most} 2+\max \left\{\phi_{\varepsilon}\left(x_{i}, x_{j}\right): 1 \leqslant i, j \leqslant n\right\}$. The proof of the second assertion is easier still and is left to the reader.

Let $l_{2}$ denote the collection of square summable real sequences made a Hilbert space in the usual way. The unit ball in this space is clearly uniformly chainable but is not totally bounded. In the same space there exists a subspace that fails to be uniformly locally compact which is not only complete but is also locally compact, sigma compact, connected, and uniformly chainable. Let $\left\{e_{i}: i \in Z^{+}\right\}$be the standard orthonormal basis for $l_{2}$. For each $i$ in $Z^{+}$define sets $Z_{i}$ and $T_{i}$ as follows:

$$
\begin{aligned}
& Z_{i}=\left\{e_{n}: n=2^{i}(2 k-1) \text { and } k \in Z^{+}\right\} \\
& T_{i}=\left\{\alpha e_{n}+(1-\alpha) e_{2 i-1}: e_{n} \in Z_{i} \text { and } 2^{-i} / \sqrt{2} \leqslant \alpha \leqslant 1\right\} .
\end{aligned}
$$

Let $L$ denote the infinite polygonal path joining $e_{1}$ to $e_{2}$, then $e_{2}$ to $e_{3}$, etc. Finally let $X=L \cup \cup_{i=1}^{\infty} T_{i}$. The subspace $X$ is connected, for it consists of the connected set $L$ and a collection of line segments each of which meets $L$. Since $X$ is a closed subspace of a Hilbert space it is complete. Moreover, $X$ is locally Euclidean; in fact, at each point $x$ in $X$ we can find a ball with center $x$ whose intersection with $X$ is either one, two, or three line segments. Thus, $X$ is locally compact. However, $X$ is not uniformly locally compact because for each $i$ the set $B_{2^{-i}}\left[e_{2 i-1}\right]$ is not compact. To see that $X$ is uniformly chainable let $\varepsilon>0$ be arbitrary. Choose $i$ such that $2^{-i}<\varepsilon$. Let $y$ and $w$ be two arbitrary points in $X$. There is a polygonal path in $X$ leading from $y$ (resp. $w$ ) to a point $y^{*}$ (resp. $w^{*}$ ) in $Z_{i}$ that consists of at most $2^{i}+1$ sides each of length at most $\sqrt{2}$. Now $y^{*}$ can be joined to $w^{*}$ by an $\varepsilon$-chain via $e_{2 i-1}$, i.e., in $T_{i}$. It follows that $y$ can be joined to $w$ by an $\varepsilon$-chain in $X$ whose length depends on $i$ and not on the choice of $y$ and $w$.

We are now ready for the main result.

TheOREM 1. Let $(X, d)$ be a chainable metric space. Then $X$ is compact if and only if $X$ is uniformly locally compact and uniformly chainable.

Proof. Since closed balls are closed sets and compactness implies total boundedness, the necessity of the conditions is immediate. To show that these conditions are sufficient choose $\delta>0$ such that all closed balls of radius $\delta$ are compact. Let $\varepsilon<\delta$ be fixed. We first show that if $C$ is a closed set, then $B_{e}[C]$ is closed. To this end let $\left\{x_{n}\right\}$ be a sequence in $B_{e}[C]$ convergent to a point $x$. For each $n$ choose $c_{n}$ in $C$ satisfying $d\left(c_{n}, x_{n}\right) \leqslant \varepsilon$. Eventually $\left\{c_{n}\right\}$ must be in $B_{\delta}[x]$ so that a subsequence of $\left\{c_{n}\right\}$ must be convergent to some point $c$ in $C$. Clearly, $d(c, x)<\varepsilon$ and therefore $B_{e}[C]$ is closed. Next let $A$ be compact. We claim that $B_{e}[A]$ is compact. Since $A$ is compact there is a finite subset $F$ of $A$ such that $A \subset B_{\delta-e}[F]$. It follows that $B_{\varepsilon}[A] \subset B_{\varepsilon}\left[B_{\delta-\varepsilon}[F]\right] \subset B_{\delta}[F]$. Thus $B_{\varepsilon}[A]$ is a closed subset of a compact set and is thus itself compact. Finally let $p$ be an arbitrary point of $X$. From the last 
arguement it follows by induction that each set $B_{e}^{n}[\{p\}]$ is compact. By hypothesis there exists an integer $n$ such that each point in $X$ can be connected to $p$ by an $\varepsilon$-chain of length at most $n$. But this means that $X=B_{\varepsilon}^{n}[\{p\}]$. Hence, $X$ is compact (and connected).

Counterexamples are in order. The intricate subspace of $l_{2}$ described earlier shows that "uniformly locally compact" cannot be replaced by "complete" and "locally compact" in the statement of Theorem 1. A much simpler example shows that "uniformly chainable" cannot be replaced by "connected" and "bounded": remetrize the real line by defining $d(x, y)=\min \{1,|x-y|\}$. We also mention that the terms "uniformly locally compact" and "uniformly chainable" were not chosen idly, for they immediately generalize to Hausdorff uniform spaces, and Theorem 1 holds in this more general context.

Compact and totally bounded spaces admit sequential characterizations: $X$ is compact (resp. totally bounded) if each sequence in $X$ has a convergent (resp. Cauchy) subsequence. The chainable spaces that are uniformly chainable are sequentially characterized by the behavior that the functions $\left\{\phi_{\varepsilon}: \varepsilon>0\right\}$ exhibit when restricted to ordered pairs whose coordinates come from an appropriately chosen subsequence. Such a characterization rests on a basic theorem of combinatorics [3].

RAMSEY'S TheOREM. Let $r$ be a positive integer and let $\left\{A_{1}, A_{2}, \ldots, A_{N}\right\}$ be a partition of the r-element subsets of $Z^{+}$. Then there is an infinite subset $S$ of $Z^{+}$and $i \in\{1,2, \ldots, N\}$ such that each r-element subset of $S$ belongs to $A_{i}$.

Definition. Let $X$ be a chainable metric space and let $\varepsilon>0$. The chain distance function $\phi_{\varepsilon}$ is said to be constant on a sequence $\left\{x_{n}\right\}$ in $X$ if $\left\{\phi_{\varepsilon}\left(x_{n}, x_{m}\right): n \neq m\right\}$ consists of exactly one number. Similarly, $\phi_{e}$ is bounded on $\left\{x_{n}\right\}$ if $\left\{\phi_{e}\left(x_{n}, x_{m}\right)\right.$ : $n \neq m\}$ is a bounded set of numbers.

THEOREM 2. Let $X$ be a chainable metric space. The following are equivalent:

(a) $X$ is uniformly chainable.

(b) For each $\varepsilon>0$ every sequence $\left\{x_{n}\right\}$ in $X$ has a subsequence on which $\phi_{\varepsilon}$ is constant.

(c) For each $\varepsilon>0$ every sequence $\left\{x_{n}\right\}$ in $X$ has a subsequence on which $\phi_{\varepsilon}$ is bounded.

(d) Let $\left\{\varepsilon_{m}\right\}$ be a sequence of positive numbers convergent to zero. Each sequence $\left\{x_{n}\right\}$ in $X$ has a subsequence $\left\{x_{n_{k}}\right\}$ such that for each $m \in Z^{+}$the function $\phi_{e_{m}}$ is constant on a tail of $\left\{x_{n_{k}}\right\}$.

Proof. (a) $\rightarrow$ (b). Let $\varepsilon>0$. By assumption there exists $N \in Z^{+}$such that for each $x$ and $y$ in $X$ we have $\phi_{\varepsilon}(x, y) \leqslant N$. If $\left\{x_{n}: n \in Z^{+}\right\}$is a finite set, then $\left\{x_{n}\right\}$ has a constant subsequence on which $\phi_{\varepsilon}$ is zero. Otherwise, by passing to a subsequence we can assume that the terms of $\left\{x_{n}\right\}$ are distinct. For each $i \in$ $\{1,2, \ldots, N\}$ let $A_{i}=\left\{\left\{x_{j}, x_{k}\right\}: \phi_{\varepsilon}\left(x_{j}, x_{k}\right)=i\right\}$. Clearly $\left\{A_{1}, \ldots, A_{N}\right\}$ partitions the two element subsets of the countably infinite set $\left\{x_{n}: n \in Z^{+}\right\}$. By Ramsey's Theorem there exist an $A_{i}$ and an infinite subset $S$ of $\left\{x_{n}: n \in Z^{+}\right\}$such that all of 
the two element subsets of $S$ belong to $A_{i}$. If we sequence $S$ in the order of the subscripts inherited from the original sequence, then we obtain the desired subsequence of $\left\{x_{n}\right\}$.

(b) $\rightarrow$ (d). According to (b) for each $m$ in $Z^{+}$we can inductively construct subsequences $\left\{x_{n}^{m}\right\}$ of $\left\{x_{n}\right\}$ such that for each $m$ (i) $\left\{x_{n}^{m+1}\right\}$ is a subsequence of $\left\{x_{n}^{m}\right\}$, and (ii) $\phi_{\varepsilon_{m}}$ is constant on $\left\{x_{n}^{m}\right\}$. For each $m$ let $x_{n_{m}}=x_{m}^{m}$. Clearly $\phi_{e_{m}}$ is constant on $x_{m}^{m}, x_{m+1}^{m+1}, x_{m+2}^{m+2}, \ldots$ which is a tail of $\left\{x_{n_{m}}\right\}$.

(d) $\rightarrow$ (a). Suppose $X$ is not uniformly chainable. Let $\left\{\varepsilon_{m}\right\}$ be a sequence of positive numbers convergent to zero. Since $I=\{\varepsilon: X$ is not uniformly $\varepsilon$-chainable $\}$ is a nondegenerate interval with left endpoint zero, there exists $m$ such that $\varepsilon_{m} \in I$. Fix $x_{0}$ in $X$. For each $n \in Z^{+}, B_{e_{m}}^{n}\left[\left\{x_{0}\right\}\right]$ must be a proper subset of $B_{e_{m}}^{n+1}\left[\left\{x_{0}\right\}\right]$ because $X$ is $\varepsilon_{m}$-chainable but not uniformly $\varepsilon_{m}$-chainable. For each $n$ choose $x_{n}$ in $B_{\varepsilon_{m}}^{n+1}\left[\left\{x_{0}\right\}\right] \backslash B_{\varepsilon_{m}}^{n}\left[\left\{x_{0}\right\}\right]$. Clearly $\phi_{\varepsilon_{m}}\left(x_{k}, x_{n}\right)>|n-k|$. Hence, $\phi_{\varepsilon_{m}}$ fails to be constant on any subsequence of $\left\{x_{n}\right\}$.

(b) $\rightarrow$ (c). Trivial.

(c) $\rightarrow$ (a). The proof is a reiteration of the proof of the implication (d) $\rightarrow$ (a).

We observe that the statement " $\left\{x_{n}\right\}$ has a Cauchy subsequence" is equivalent to a stronger form of condition (d): Let $\left\{\varepsilon_{m}\right\}$ be a sequence of positive numbers convergent to zero, and let $\left\{x_{n}\right\}$ be a sequence in $X$. If $\left\{x_{n}\right\}$ has no constant subsequence, then $\left\{x_{n}\right\}$ has a subsequence $\left\{x_{n_{k}}\right\}$ such that for each $m$ the function $\phi_{e_{m}}$ is one on a tail of $\left\{x_{n_{k}}\right\}$.

We note that the bounded metric spaces are precisely those that can be isometrically imbedded in complete uniformly chainable spaces. Since uniformly chainable spaces are bounded, the sufficiency of the condition is clear. On the other hand since a bounded metric space $X$ can be imbedded in a closed ball of the Banach space $C(X)$ of bounded continuous real valued functions on $X$ [4], the condition is also necessary. Next we give a rather curious characterization of the uniformly chainable spaces among the bounded chainable ones. Let $X$ be a bounded chainable metric space. If $A \subset X$ is nonempty we define the distance $\delta$ from $A$ to $X$ by the formula

$$
\delta(A, X)=\sup _{x \in X} d(x, A) .
$$

Of course $\delta$ just gives the Hausdorff distance from $A$ to $X$ [1]. For each $n$ in $Z^{+}$let $f_{\varepsilon}^{n}(A)=\delta\left(B_{\varepsilon}^{n}[A], X\right)$. Notice that $\left\{f_{\varepsilon}^{n}(A)\right\}$ is a decreasing sequence of nonnegative reals and thus converges (though not necessarily to zero). A nontrivial characterization is determined by the rate of convergence over all subsets $A$ of $X$.

THEOREM 3. Let $X$ be a bounded chainable metric space. If $A$ is a nonempty subset of $X$ let $f_{\varepsilon}^{n}(A)$ denote the Hausdorff distance from $B_{\varepsilon}^{n}[A]$ to $X$. Then $X$ is uniformly chainable iff, for each $\varepsilon>0,\left\{f_{\varepsilon}^{n}\right\}$ converges uniformly on the set of nonempty subsets of $X$.

Proof. Let $X$ be uniformly chainable. If $\varepsilon>0$ there exists $n \in Z^{+}$such that each two points in $X$ can be joined by an $\varepsilon$-chain of length at most $n$. It follows that $f_{e}^{k}(A)=0$ for each nonemtpy set $A$ and $k \geqslant n$. Conversely suppose that $X$ is 
not uniformly $\varepsilon$-chainable for some positive $\varepsilon$. Fix $x_{0}$ in $X$. Then for each $n$ in $Z^{+}$, $X \backslash B_{\varepsilon}^{n}\left[\left\{x_{0}\right\}\right] \neq \varnothing$. Fix $n$ and let $A=\left\{x_{0}\right\} \cup X \backslash B_{\varepsilon}^{2 n+4}\left[\left\{x_{0}\right\}\right]$. For each $k>2 n+$ 4 we have $f_{\varepsilon}^{k}(A)=0$. On the other hand it is easy to see that

$$
B_{e}^{n}[A] \subset B_{e}^{n}\left[\left\{x_{0}\right\}\right] \cup\left(X \backslash B_{e}^{n+4}\left[\left\{x_{0}\right\}\right]\right) \text {. }
$$

As a result each point in the nonempty set $B_{\varepsilon}^{n+2}\left[\left\{x_{0}\right\}\right] \backslash B_{\varepsilon}^{n+1}\left[\left\{x_{0}\right\}\right]$ has distance in excess of $\varepsilon$ from $B_{\varepsilon}^{n}[A]$ so that $f_{\varepsilon}^{n}(A)>\varepsilon$. Since $n$ was arbitrary $\left\{f_{\varepsilon}^{n}\right\}$ fails to converge uniformly.

\section{REFERENCES}

1. J. Dugundji, Topology, Allyn and Bacon, Boston, Mass., 1966.

2. M. H. A. Newman, Elements of topology of plane sets of points, Cambridge Univ. Press, New York, 1961.

3. F. Ramsey, On a problem of formal logic, Proc. London Math. Soc. (2) 30 (1930), 264-286.

4. G. Simmons, Introduction to topology and modern analysis, McGraw-Hill, New York, 1963.

Department of Mathematics, California State University, los Angeles, California 90032 